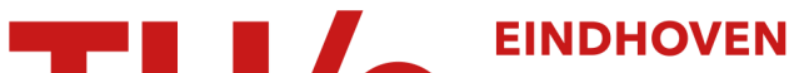

\section{Hybrid superconductor-quantum point contact devices using InSb nanowires}

Citation for published version (APA):

Gill, S. T., Damasco, J., Car, D., Bakkers, E. P. A. M., \& Mason, N. (2016). Hybrid superconductor-quantum point contact devices using InSb nanowires. Applied Physics Letters, 109(23), [233502 ].

https://doi.org/10.1063/1.4971394

DOI:

10.1063/1.4971394

Document status and date:

Published: 05/12/2016

Document Version:

Publisher's PDF, also known as Version of Record (includes final page, issue and volume numbers)

Please check the document version of this publication:

- A submitted manuscript is the version of the article upon submission and before peer-review. There can be important differences between the submitted version and the official published version of record. People interested in the research are advised to contact the author for the final version of the publication, or visit the $\mathrm{DOI}$ to the publisher's website.

- The final author version and the galley proof are versions of the publication after peer review.

- The final published version features the final layout of the paper including the volume, issue and page numbers.

Link to publication

\section{General rights}

Copyright and moral rights for the publications made accessible in the public portal are retained by the authors and/or other copyright owners and it is a condition of accessing publications that users recognise and abide by the legal requirements associated with these rights.

- Users may download and print one copy of any publication from the public portal for the purpose of private study or research.

- You may not further distribute the material or use it for any profit-making activity or commercial gain

- You may freely distribute the URL identifying the publication in the public portal.

If the publication is distributed under the terms of Article $25 \mathrm{fa}$ of the Dutch Copyright Act, indicated by the "Taverne" license above, please follow below link for the End User Agreement:

www.tue.nl/taverne

Take down policy

If you believe that this document breaches copyright please contact us at:

openaccess@tue.nl

providing details and we will investigate your claim. 


\section{Hybrid superconductor-quantum point contact devices using InSb nanowires}

S. T. Gill, J. Damasco, D. Car, E. P. A. M. Bakkers, and N. Mason

Citation: Appl. Phys. Lett. 109, 233502 (2016); doi: 10.1063/1.4971394

View online: http://dx.doi.org/10.1063/1.4971394

View Table of Contents: http://aip.scitation.org/toc/apl/109/23

Published by the American Institute of Physics

\section{Articles you may be interested in}

Accessing Rashba states in electrostatically gated topological insulator devices

Appl. Phys. Lett. 109, 232408232408 (2016); 10.1063/1.4971834

Interplay between quantum well width and interface roughness for electron transport mobility in GaAs quantum wells

Appl. Phys. Lett. 109, 232105232105 (2016); 10.1063/1.4971824

Ballistic one-dimensional transport in InAs nanowires monolithically integrated on silicon

Appl. Phys. Lett. 110, 083105083105 (2017); 10.1063/1.4977031

Spin transport in nanoscale Si-based spin-valve devices

Appl. Phys. Lett. 109, 232402232402 (2016); 10.1063/1.4971351

Improved interface properties of GaN metal-oxide-semiconductor device with non-polar plane and AIN passivation layer

Appl. Phys. Lett. 109, 232101232101 (2016); 10.1063/1.4971352

Tunnel electroresistance in $\mathrm{BiFeO} 3$ junctions: size does matter

Appl. Phys. Lett. 109, 232902232902 (2016); 10.1063/1.4971311

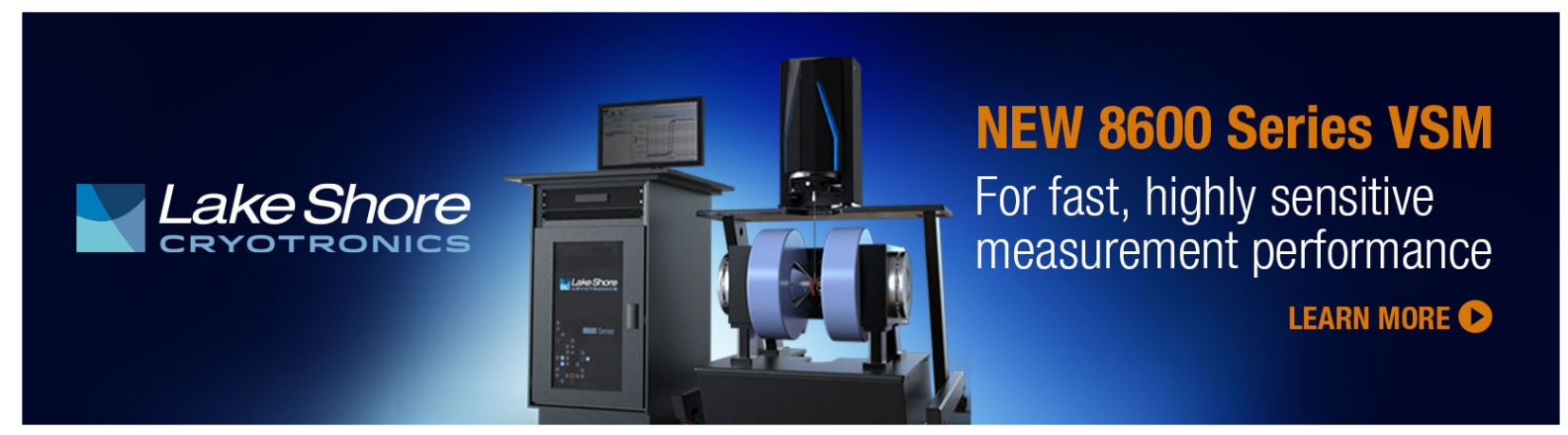




\title{
Hybrid superconductor-quantum point contact devices using InSb nanowires
}

\author{
S. T. Gill, ${ }^{1}$ J. Damasco, ${ }^{1}$ D. Car, ${ }^{2}$ E. P. A. M. Bakkers, ${ }^{2,3,4}$ and N. Mason ${ }^{1}$ \\ ${ }^{1}$ Department of Physics, University of Illinois at Urbana-Champaign, Urbana, Illinois 61801, USA \\ ${ }^{2}$ Department of Applied Physics, Eindhoven University of Technology, $5600 \mathrm{MB}$ Eindhoven, The Netherlands \\ ${ }^{3}$ QuTech, Delft University of Technology, 2600 GA Delft, The Netherlands \\ ${ }^{4}$ Kavli Institute of Nanoscience, Delft University of Technology, 2600 GA Delft, The Netherlands
}

(Received 8 October 2016; accepted 21 November 2016; published online 5 December 2016)

\begin{abstract}
Proposals for studying topological superconductivity and Majorana bound states in a nanowire proximity coupled to superconductors require that transport in the nanowire is ballistic. Previous works on hybrid nanowire-superconductor systems have shown evidence for Majorana bound states, but these experiments were also marked by disorder, which disrupts ballistic transport. In this paper, we demonstrate ballistic transport in the InSb nanowires interfaced directly with superconducting $\mathrm{Al}$ by observing quantized conductance at zero-magnetic field. Additionally, we demonstrate that the nanowire is proximity coupled to the superconducting contacts by observing Andreev reflection. These results are important steps for robustly establishing topological superconductivity in the InSb nanowires. Published by AIP Publishing.
\end{abstract}

[http://dx.doi.org/10.1063/1.4971394]

The InAs and InSb nanowires (NWs) coupled to superconductors are promising material candidates for studying topological superconductivity harboring Majorana bound states $^{1,2}$ and demonstrating non-Abelian particle statistics relevant for topological quantum computation. ${ }^{3}$ The basic procedure to observe Majorana zero modes involves tuning a superconducting proximity coupled quantum wire (i.e., a ballistic 1D system) with strong spin-orbit coupling and one spin degenerate mode in magnetic field. ${ }^{1}$ Indeed, evidence for Majorana bound states has been observed in proximitycoupled InSb and InAs NWs as a zero-bias conduction peak in tunneling experiments. ${ }^{4-7}$ However, the preliminary experiments probing Majorana bound states in nanowires were marked by disorder and a soft superconducting gap in the tunneling regime. ${ }^{4-8}$ Disorder in nanowire systems is known to break up ballistic transport, ${ }^{9,10}$ which is a crucial ingredient for developing 1D topological superconductivity. $^{1,2,4,9}$ Additionally, disorder can produce zero-bias conductance signatures similar to the Majorana bound states. ${ }^{11}$ While the typical signature of ballistic 1D transport-quantized conductance- - has been observed in InSb nanowires at high magnetic field ${ }^{9}$ and more recently at zero-field, ${ }^{19}$ clear demonstrations of ballistic transport at lower fields $(<1 \mathrm{~T}$, i.e., before the expected onset of topological superconductivity) in hybrid nanowire-superconductor systems are lacking. Hence, in order to clearly demonstrate topological superconductivity and remove alternative mechanisms for observing zero bias conduction peaks, quantized conductance should be observed at zero magnetic field in NW proximity coupled to superconductors.

Quantized conductance at zero-magnetic field is the step-like increase in conductance with gate voltage through a ballistic $1 \mathrm{D}$ constriction, in units of the quantum of conductance, $G_{O}=2 \mathrm{e}^{2} / h$, for each spin degenerate subband. ${ }^{12}$ While quantized conductance in quantum point contacts (QPCs) defined on 2D electron gas (2DEG) materials, such as GaAs heterostructures, is well established, ${ }^{12-14}$ the demonstrations of quantized conductance in nanowire systems are sparse. In contrast to QPCs in 2DEGs, short nanowire channels contacted by metals are more prone to backscattering effects. For example, typical processing of the nanowire-metal interface requires etching, which can lead to large structural disorder and hence a high probability of backscattering. ${ }^{9,10}$ In addition, the mobility in nanowires can be strongly impacted by adsorbates on the uncontacted area. ${ }^{15} \mathrm{~A}$ recent advance in nanowire synthesis has produced epitaxy of superconducting aluminum to InAs nanowires, ${ }^{16,17}$ but residual disorder in the InAs nanowire results in unintentional quantum confined regions in these wires. ${ }^{17}$ InSb NWs, in contrast, have higher electron mobility ${ }^{9,15,18}$ and, as evidenced by clear demonstrations of quantized conductance, ${ }^{10,19}$ less intrinsic disorder than InAs NWs. InSb NWs also have large Lande $\mathrm{g}$ factors of $\sim 40$ to $50,{ }^{19}$ compared to the values of $\sim 5$ to 10 in InAs; ${ }^{20-22}$ this allows for lower magnetic fields required to induce topological superconductivity. ${ }^{1-4}$ With optimized superconductor-nanowire interfaces, InSb NWs could be potentially a robust platform for observing and controlling 1D topological superconductivity.

In this work, we report on the development of $1 \mathrm{D}$ ballistic transport in InSb nanowires contacted by superconducting Al. Disorder in the nanowire is minimized by careful etching of the native oxide formed on the InSb. The low-disorder at the interface enables the hybrid superconductor-nanowire devices to behave as quantum point contacts (QPC), where we observe quantized conductance at zero-magnetic field. Additionally, we find that the level spacing of subbands in InSb nanowires differs from that seen in conventional $2 \mathrm{DEG}$ QPCs. In particular, we find evidence of near degeneracy in the energy spacing of the 2nd-3rd and 4th-5th subbands. Finally, we demonstrate proximity effect of the superconducting contacts by observing the gate-tunable Andreev reflection. 

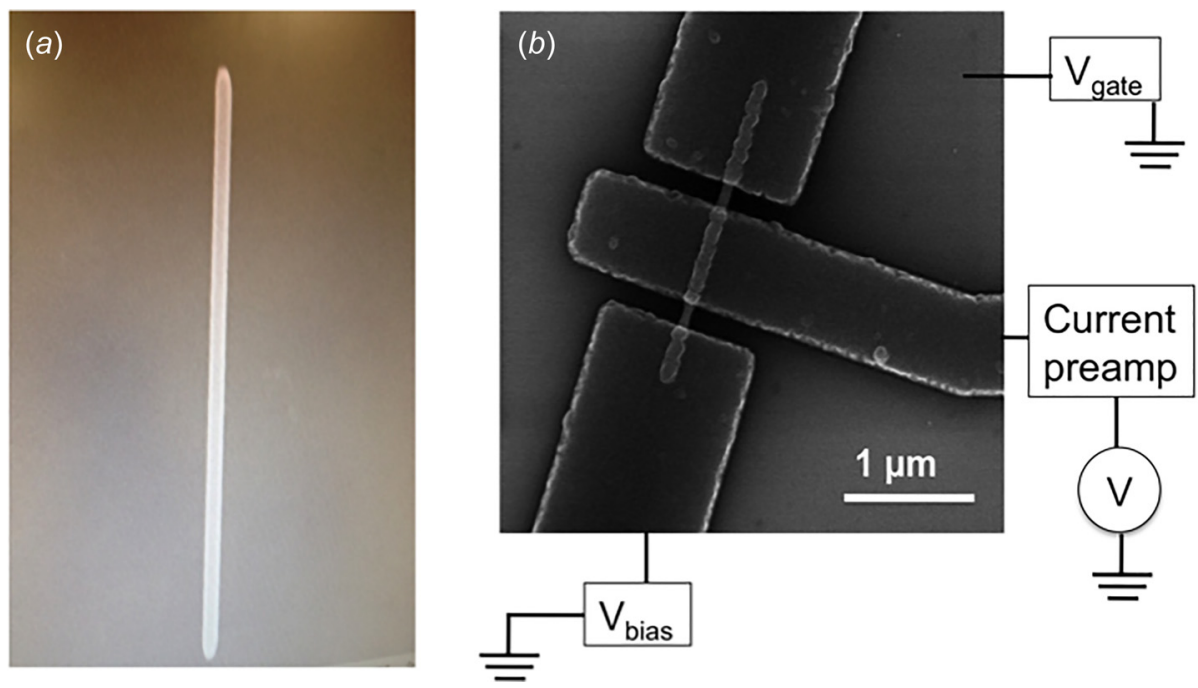

FIG. 1. (a) SEM image of a $2 \mu \mathrm{m}$ long $\mathrm{InSb}$ nanowire that has been directly interfaced with $15 \mathrm{~nm}$ of $\mathrm{Al}$. The Al shell is uniform along the entire nanowire and no grains or roughness can be observed given the resolution of the SEM. (b) SEM image with a schematic of the measurement circuit for a completed $\mathrm{InSb}$ nanowire device on an $\mathrm{Si} /$ $\mathrm{SiO}_{2}$ chip contacted by $20 \mathrm{~nm} \mathrm{Al} / 5 \mathrm{~nm}$ $\mathrm{Ti} / 120 \mathrm{~nm} \mathrm{Al}$.

To make the devices, we use InSb nanowires $(1-3 \mu \mathrm{m}$ long, $50-80 \mathrm{~nm}$ diameter) grown by metal-organic phase epitaxy, ${ }^{18}$ which are then transferred from the growth chip by use of a micromanipulator to a pre-patterned Si chip with a $300 \mathrm{~nm}$ silicon dioxide layer serving as a gate dielectric. The chip is cleaned using reactive ion etching prior to nanowire deposition to remove resist polymers that can degrade nanowire transport. Contacts to the nanowires are defined by electron beam lithography. Prior to metal deposition, the native oxide formed on the nanowire is removed by sulfur passivation. ${ }^{23}$ Previous works on InSb nanowires had prepared contacts by etching the native oxide using ion milling. . $^{40,15,18}$ This procedure effectively removes the oxide to make electrical contacts, but the milling is generally harsh to the InSb crystal. ${ }^{24}$ The homogenous etching of the InSb nanowire enabled by sulfur passivation allows deposition of thin films $(<25 \mathrm{~nm})$ of aluminum having a uniform morphology along the nanowire, as shown in Figure 1(a). In order to prevent surface reconstruction of the $\mathrm{Al}$ interfacing the $\mathrm{InSb},{ }^{16}$ we evaporate $5-10 \mathrm{~nm}$ of $\mathrm{Ti}$ in between a final layer of $\mathrm{Al}$ for our contacts. Figure 1(b) shows an SEM image of a completed device where the $\mathrm{InSb}$ nanowire is contacted by an $\mathrm{Al} / \mathrm{Ti} / \mathrm{Al}$ $(20 / 5-10 / 120 \mathrm{~nm})$ trilayer. Previous characterization of similarly grown InSb nanowires provided an extracted mean free path of $300 \mathrm{~nm} .{ }^{9,18}$ The contact spacing for the InSb nanowire/superconductor QPCs is $\mathrm{L}=150-300 \mathrm{~nm}$, so the channel length is comparable to or smaller than the mean free path of the nanowire. Nanowire devices are wirebonded immediately after liftoff and left in vacuum ( $\left.\leq 10^{-2} \mathrm{mBar}\right)$ for $24-48 \mathrm{~h}$ to remove adsorbates before measurement in a dilution fridge.

Figure 2(a) (left) depicts the typical spin degenerate 1D subbands for a gate-defined QPC (e.g., in GaAs 2DEGs). As the Fermi energy is tuned through the subbands with a gate voltage, the resultant conductance at zero dc bias is staircaselike, as depicted in Figure 2(b) (left), where conductance increases with gate voltage in approximately even steps of $G_{\mathrm{o}}=2 \mathrm{e}^{2} / \mathrm{h}$. In contrast, $1 \mathrm{D}$ constrictions with rotational symmetry, such as nanowires and nanotubes, possess a different subband structure, which results in different conductance quantization..$^{19,25-27}$ Rotational symmetry of the nanowire should result in a total or near degeneracy of the 2nd-3rd and 4th-5th subbands. ${ }^{19,26,27}$ Figure 2(c) shows conductance vs gate voltage measurements for a nanowire QPC having a channel length of $150 \mathrm{~nm}$ at $10 \mathrm{mV}$ applied bias. The nanowire conductance can be understood by considering the $B=0$ subband structure depicted in Figure 2(a) (right), which produces conductance signatures consistent with what we observe in the data (depicted in Figure 2(b), right) for high bias. The data are shown for magnetic fields $B=0,1$, and $2 \mathrm{~T}$, where each sweep is offset by $+5 \mathrm{~V}$ in the plot respectively. At $B=0$ in Figure 2(c), the QPC exhibits plateaus at $1 G_{0}$, $2.5 G_{0}$, and $5 G_{0}$. In some of the zero-field data, as shown in Figure 2(c), a plateau at $2.5 G_{0}$ is observed at finite bias as opposed to $3 G_{0}$. A "half plateau" at finite bias indicates there is a difference of one subband between the source and drain electrodes. Assuming the subbands are spin-degenerate, the plateau at $2.5 G_{0}$ indicates a small but finite energy separation between the 2 nd and 3rd subbands, consistent with the
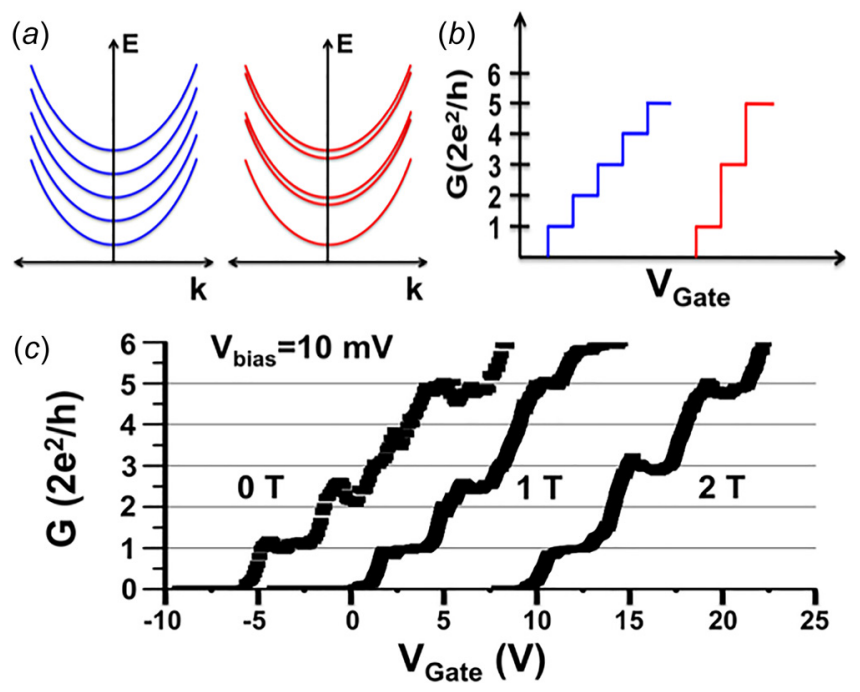

FIG. 2. (a) Diagram of the 1D subband dispersion relation for a gate defined QPC (left, in blue) and a nanowire with rotational symmetry (right, in red). (b) Expected quantized conductance for a gate defined QPC (left, in blue) and for a rotationally symmetric QPC (right, in red) having degeneracy of the 2nd-3rd and 4th-5th subbands. (c) Gate dependence of conductance at $10 \mathrm{mV}$ bias taken at a temperature of $20 \mathrm{mK}$ and for magnetic field perpendicular to the substrate. As magnetic field is increased, subband crossing creates a degeneracy of the 2nd and 3rd subbands that produces conductance quantization identical with the conductance quantization shown on the right in (b) for a rotationally symmetric QPC having degenerate subbands. 

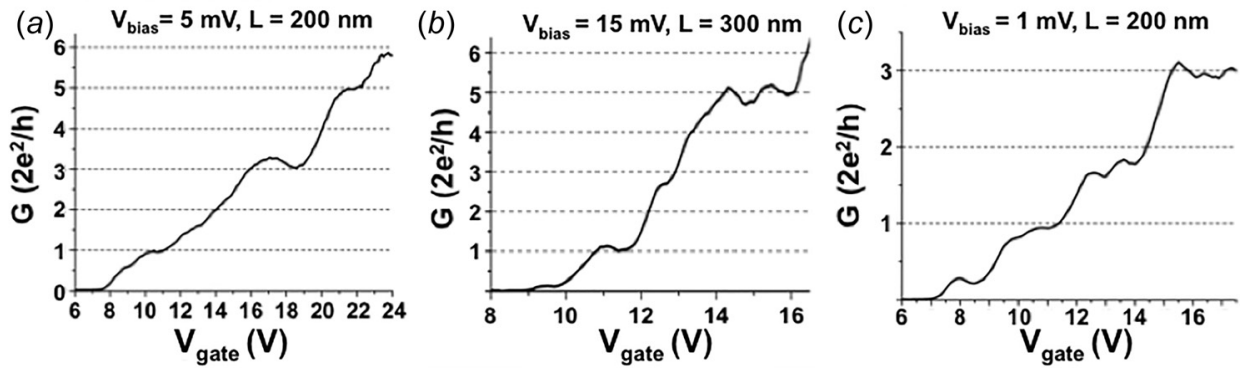

FIG. 3. (a) and (b) Conductance quantization at zero magnetic field for InSb QPCs having channel lengths of $200 \mathrm{~nm}$ and $300 \mathrm{~nm}$, respectively. The conductance quantization is consistent with that for a QPC having rotational symmetry. (c) At small bias $\left(\mathrm{V}_{\text {bias }}=1 \mathrm{mV}\right.$ in this case), a plateau roughly at $2 G_{0}$ can be resolved. The $2 G_{0}$ plateau indicates that the 2 nd and 3rd subbands are not degenerate. subbands plotted in Figure 2(a) (right). As we increase magnetic field, the high bias plateau at $2.5 G_{0}$ evolves to $3 G_{O}$ at $B=2$ Tesla without additional plateaus emerging. This evolution implies that degeneracy from crossings between subbands 2 and 3 occurs with increasing magnetic field. Similar plateau structure and evolution in magnetic field have recently been reported in InSb nanowire QPCs contacted by nonsuperconducting chromium/gold. ${ }^{19}$ Additionally, numerical simulations also support a degeneracy from crossing between subbands 2 and 3 of InSb nanowires emerging around $2 \mathrm{~T}{ }^{19}$

We observe conductance quantization consistent with a QPC having rotational symmetry in all our InSb QPC devices measured at high bias. Figures 3(a) and 3(b) show the zero-magnetic field conductance quantization for two different length QPCs. Both of these devices show quantized conductance at high bias consistent with the subband spacing discussed previously. Figure 3(c) shows the gate dependence of the conductance for the $200 \mathrm{~nm}$ length QPC from Figure 3(a) taken at $1 \mathrm{mV}$ bias, which is a substantially smaller bias than that for the data shown in Figures 3(a) and 3(b). In this scan, a plateau at roughly $2 G_{0}$ appears between plateaus at 1 and $3 G_{O}$. The imperfect quantization of the $2 G_{O}$ plateau is likely related to remaining disorder in the device. ${ }^{19}$ The plateau emerging near $2 G_{0}$ indicates there is a finite energy difference in the spacing of the 2 nd and 3 rd subband that is not resolved for high bias scans.

Finally, we discuss the proximity effect of the superconducting leads to the InSb nanowire. As shown in Figure 4(a), we observe a gate tunable modulation of conduction centered between $\mathrm{V}_{\mathrm{SD}} \approx \pm 250 \mu \mathrm{V}$. Similar modulation is seen in all our devices. For larger gate voltages (i.e., higher conductance values), the modulation develops into clearly distinguishable peaks at $\mathrm{V}_{\mathrm{SD}} \approx \pm 250 \mu \mathrm{V}$. The peaks occur at a bias consistent with twice the induced gap commonly seen for Ti/Al leads $\left(\Delta_{0} \approx 125 \mu \mathrm{eV}\right)$. Figure 4(b) shows the behavior of the differential conductance peaks near zero-bias as a function of temperature. As expected for Andreev reflection, the peaks vanish as the temperature is increased towards the superconducting transition temperature of the Al leads $\left(T_{c} \sim 1 \mathrm{~K}\right)$. Also, the conduction peaks nearly double in magnitude below $100 \mathrm{mK}$, consistent with the predictions for Andreev reflection. ${ }^{28}$ These observations establish that the NWs are proximity coupled to superconducting leads. We note that we do not detect supercurrents across the device. Previous works on superconductivity in few mode 1D materials have consistently demonstrated small supercurrents. $^{29-32}$ The absence of clear supercurrents in our samples is likely because of an elevated electron temperature of the leads. ${ }^{33}$ By employing a similar electronic filtering setup to the previous studies of nanowire Josephson junctions, ${ }^{30,32}$ we should be able to achieve strong correlations between conductance steps and supercurrents.

The ability to realize ballistic transport and proximity coupled superconductivity in InSb NWs is an important step for robustly developing Majorana bound states in NWs. Since we observe quantized conductance plateaus at $G=G_{0}$, we can establish that the hybrid superconductor-InSb NW QPC devices are tuned to the 1st mode quantum wire regime. Additionally, the superconducting contacts can be modified for high magnetic field compatibility by using an $\mathrm{Al} / \mathrm{NbTi} /$ NbTiN trilayer. Hence, the procedure we have developed for making hybrid superconductor QPC devices using InSb nanowires satisfies the generic requirements for creating Majorana modes.

In conclusion, we have observed quantized conductance in InSb NWs directly interfaced with a thin layer of superconducting $\mathrm{Al}$ at zero magnetic field. We demonstrated that the subbands in the wire are not uniformly spaced and that there is a near degeneracy in the dispersion relation of the second-third and fourth-fifth subbands. In addition to realizing ballistic transport in the wires, we demonstrated the proximity effect of the superconducting contacts by
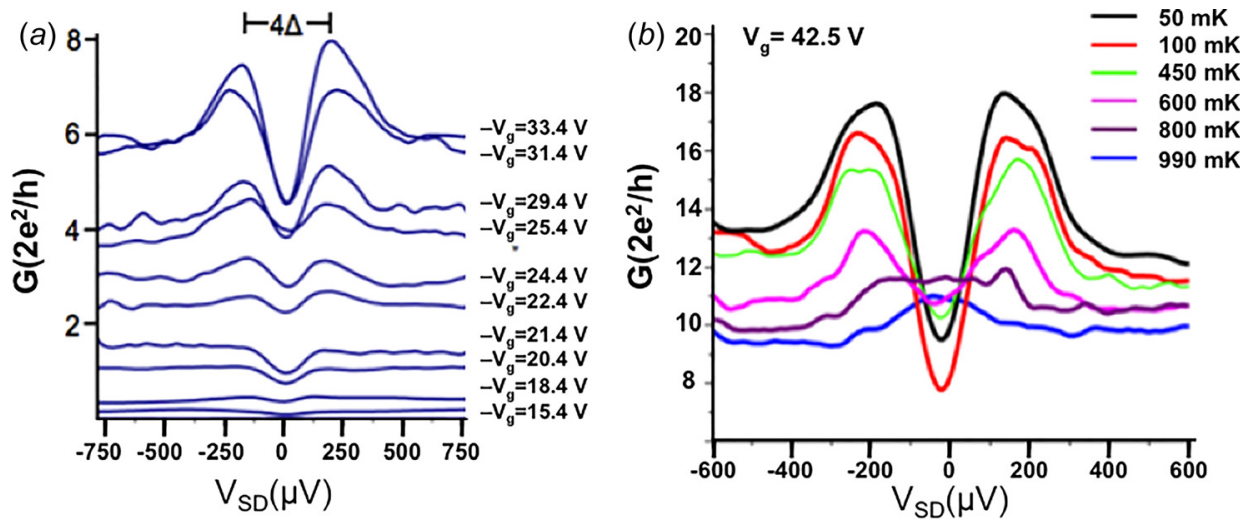

FIG. 4. (a) Differential conductance as a function of source-drain bias is plotted for different gate voltage values. Conductance is modulated around $\mathrm{V}_{\mathrm{SD}}=250 \mu \mathrm{V}$. This enhancement occurs at a value consistent with twice the induced gap and is a signature of Andreev reflection. (b) Temperature dependence of the conductance enhancement from Andreev reflection. As the sample temperature approaches the $\mathrm{T}_{\mathrm{C}}$ of $\mathrm{Al}$, the conductance peaks thermally broaden into a single peak that nearly vanishes at $T=990 \mathrm{mK}$. 
observing Andreev reflection. By studying similar hybrid NW-Superconductor QPC devices at lower electron temperature, we anticipate the devices should show rich interplay between ballistic transport and superconductivity.

This work was supported by the Office of Naval Research Grant No. N0014-16-1-2270. Device fabrication was done in the Materials Research Lab Central Facilities at the University of Illinois.

${ }^{1}$ Y. Oreg, G. Refael, and F. von Oppen, Phys. Rev. Lett. 105, 177002 (2010). ${ }^{2}$ R. M. Lutchyn, J. D. Sau, and S. D. Sarma, Phys. Rev. Lett. 105, 077001 (2010).

${ }^{3}$ J. Alicea, Y. Oreg, G. Refael, F. von Oppen, and M. P. A. Fisher, Nat. Phys. 7, 412 (2011).

${ }^{4}$ V. Mourik, K. Zuo, S. M. Frolov, S. R. Plissard, E. Bakkers, and L. P. Kouwenhoven, Science 336, 1003 (2012).

${ }^{5}$ A. Das, Y. Ronen, Y. Most, Y. Oreg, M. Heiblum, and H. Shtrikman, Nat. Phys. 8, 887 (2012)

${ }^{6}$ M. T. Deng, C. L. Yu, G. Y. Huang, M. Larsson, P. Caroff, and H. Q. Xu, Nano Lett. 12, 6414 (2012).

${ }^{7}$ H. O. H. Churchill, V. Fatemi, K. Grove-Rasmussen, M. T. Deng, P. Caroff, H. Q. Xu, and C. M. Marcus, Phys. Rev. B 87, 241401 (2013).

${ }^{8}$ S. Takei, B. M. Fregoso, H. Y. Hui, A. M. Hui, and S. Das Sarma, Phys. Rev. Lett. 110, 186803 (2013).

${ }^{9}$ I. van Weperen, S. R. Plissard, E. P. A. M. Bakkers, S. M. Frolov, and L. P. Kouwenhoven, Nano Lett. 13, 387 (2013).

${ }^{10}$ A. V. Kretinin, R. Popovitz-Biro, D. Mahalu, and H. Shtrikman, Nano Lett. 10, 3439 (2010).

${ }^{11}$ J. Liu, A. C. Potter, K. T. Law, and P. A. Lee, Phys. Rev. Lett. 109, 267002 (2012).

${ }^{12}$ B. J. Van Wees, H. Van Houten, C. W. J. Beenakker, J. G. Williamson, L. P. Kouwenhoven, D. Van der Marel, and C. T. Foxon, Phys. Rev. Lett. 60, 848 (1988).

${ }^{13}$ D. A. Wharam, T. J. Thornton, R. Newbury, M. Pepper, H. Ahmed, J. E. F. Frost, D. G. Hasko, D. C. Peacock, D. A. Ritchie, and G. A. C. Jones, J. Phys. C 21, L209 (1988).

${ }^{14}$ S. M. Cronenwett, H. J. Lynch, D. Goldhaber-Gordon, L. P. Kouwenhoven, C. M. Marcus, K. Hirose, N. S. Wingreen, and V. Umansky, Phys. Rev. Lett. 88, 226805 (2002).
${ }^{15}$ Ö. Gül, D. J. van Woerkon, I. van Weperen, D. Car, S. R. Plissard, E. P. A. M. Bakkers, and L. P. Kouwenhoven, Nanotechnology 26, 215202 (2015).

${ }^{16}$ P. Krogstrup, N. L. B. Ziino, S. M. Albrecht, M. H. Madsen, E. Johnson, J. Nygard, C. M. Marcus, and T. S. Jespersen, Nat. Mater. 14, 400 (2015).

${ }^{17}$ W. Chang, S. M. Albrecht, T. S. Jespersen, F. Kuemmeth, P. Krogstrup, J. Nygard, and C. M. Marcus, Nat. Nanotechnol. 10, 232 (2015).

${ }^{18}$ S. R. Plissard, D. R. Slapak, M. A. Verheijen, M. Hocevar, G. G. W. Immin, I. Van Weperen, S. Nadj-Perge, S. M. Frolov, L. P. Kouwenhoven, and E. P. A. M. Bakkers, Nano Lett. 12, 1794 (2012).

${ }^{19}$ J. Kammhuber, M. C. Cassidy, H. Zhang, Ö. Gül, F. Pei, M. W. A. de Moor, B. Nijholt, K. Watanabe, T. Taniguchi, D. Car, S. R. Plissard, E. P. A. M. Bakkers, and L. P. Kouwenhoven, Nano Lett. 16, 3482 (2016).

${ }^{20}$ S. Csonka, L. Hofstetter, F. Freitag, S. Oberholzer, C. Schönenberger, T. S. Jespersen, M. Aagesen, and J. Nygård, Nano Lett. 8, 3932 (2008).

${ }^{21}$ M. D. Schroer, K. D. Petersson, M. Jung, and J. R. Petta, Phys. Rev. Lett. 107, 176811 (2011).

${ }^{22}$ E. J. H. Lee, X. Jiang, M. Houzet, R. Aguado, C. M. Lieber, and S. De Franceschi, Nat. Nanotechnol. 9, 79 (2014).

${ }^{23}$ D. B. Suyatin, C. Thelander, M. T. Björk, I. Maximov, and L. Samuelson, Nanotechnology 18, 105307 (2007).

${ }^{24}$ V. Mourik and K. Zou, Ph.D. thesis, TU Delft, 2016.

${ }^{25}$ M. J. Biercuk, N. Mason, J. Martin, A. Yacoby, and C. M. Marcus, Phys. Rev. Lett. 94, 026801 (2005).

${ }^{26}$ A. Ford, S. B. Kumar, R. Kapadia, J. Guo, and A. Javey, Nano Lett. 12, 1340 (2012)

${ }^{27}$ J. M. Krans, J. M. Van Ruitenbeek, V. V. Fisun, I. K. Yanson, and L. J. De Jongh, Nature 375, 767 (1995).

${ }^{28}$ G. E. Blonder, M. Tinkham, and T. M. Klapwijk, Phys. Rev. B 25, 4515 (1982).

${ }^{29}$ P. Jarillo-Herrero, J. A. Van Dam, and L. P. Kouwenhoven, Nature 439, 953 (2006).

${ }^{30}$ J. Xiang, A. Vidan, M. Tinkham, R. M. Westervelt, and C. M. Lieber, Nat. Nanotechnol. 1, 208 (2006).

${ }^{31}$ J. P. Cleuziou, W. Wernsdorfer, V. Bouchiat, T. Ondarçuhu, and M. Monthioux, Nat. Nanotechnol. 1, 53 (2006).

${ }^{32}$ Y. J. Doh, J. A. van Dam, A. L. Roest, E. P. A. M. Bakkers, L. P. Kouwenhoven, and S. De Francheschi, Science 309, 272 (2005).

${ }^{33}$ D. Vion, M. Gotz, P. Joyez, D. Esteve, and M. H. Devoret, Phys. Rev. Lett. 77, 3435 (1996). 